\title{
Antimicrobial-specific response from resistance gene carriers studied in a natural, highly diverse microbiome
}

\author{
Wisnu Adi Wicaksono ${ }^{1}$, Peter Kusstatscher', Sabine Erschen', Tamara Reisenhofer-Graber ${ }^{1}$, Martin Grube², \\ Tomislav Cernava ${ }^{1 *}$ (1) and Gabriele Berg ${ }^{1}$
}

\begin{abstract}
Background: Antimicrobial resistance (AMR) is a major threat to public health. Microorganisms equipped with AMR genes are suggested to have partially emerged from natural habitats; however, this hypothesis remains inconclusive so far. To understand the consequences of the introduction of exogenic antimicrobials into natural environments, we exposed lichen thalli of Peltigera polydactylon, which represent defined, highly diverse miniature ecosystems, to clinical (colistin, tetracycline), and non-clinical (glyphosate, alkylpyrazine) antimicrobials. We studied microbiome responses by analysing DNA- and RNA-based amplicon libraries and metagenomic datasets.

Results: The analyzed samples consisted of the thallus-forming fungus that is associated with cyanobacteria as well as other diverse and abundant bacterial communities (up to $10^{8} 16 \mathrm{~S}$ rRNA gene copies $\mathrm{ng}^{-1}$ DNA) dominated by Alphaproteobacteria and Bacteroidetes. Moreover, the natural resistome of this meta-community encompassed 728 AMR genes spanning 30 antimicrobial classes. Following 10 days of exposure to the selected antimicrobials at four different concentrations (full therapeutic dosage and a gradient of sub-therapeutic dosages), we observed statistically significant, antimicrobial-specific shifts in the structure and function but not in bacterial abundances within the microbiota. We observed a relatively lower response after the exposure to the non-clinical compared to the clinical antimicrobial compounds. Furthermore, we observed specific bacterial responders, e.g., Pseudomonas and Burkholderia to clinical antimicrobials. Interestingly, the main positive responders naturally occur in low proportions in the lichen holobiont. Moreover, metagenomic recovery of the responders' genomes suggested that they are all naturally equipped with specific genetic repertoires that allow them to thrive and bloom when exposed to antimicrobials. Of the responders, Sphingomonas, Pseudomonas, and Methylobacterium showed the highest potential.
\end{abstract}

Conclusions: Antimicrobial exposure resulted in a microbial dysbiosis due to a bloom of naturally low abundant taxa (positive responders) with specific AMR features. Overall, this study provides mechanistic insights into community-level responses of a native microbiota to antimicrobials and suggests novel strategies for AMR prediction and management.

Keywords: Lichen microbiota, Peltigera polydactylon, Antimicrobial resistance, Metagenomic mining, Genome recovery

\footnotetext{
* Correspondence: tomislav.cernava@tugraz.at

${ }^{1}$ Institute of Environmental Biotechnology, Graz University of Technology, Graz, Austria

Full list of author information is available at the end of the article
}

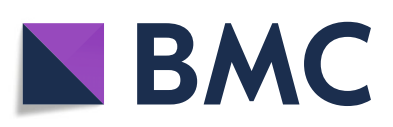

(c) The Author(s). 2021 Open Access This article is licensed under a Creative Commons Attribution 4.0 International License, which permits use, sharing, adaptation, distribution and reproduction in any medium or format, as long as you give appropriate credit to the original author(s) and the source, provide a link to the Creative Commons licence, and indicate if changes were made. The images or other third party material in this article are included in the article's Creative Commons licence, unless indicated otherwise in a credit line to the material. If material is not included in the article's Creative Commons licence and your intended use is not permitted by statutory regulation or exceeds the permitted use, you will need to obtain permission directly from the copyright holder. To view a copy of this licence, visit http://creativecommons.org/licenses/by/4.0/ The Creative Commons Public Domain Dedication waiver (http://creativecommons.org/publicdomain/zero/1.0/) applies to the data made available in this article, unless otherwise stated in a credit line to the data. 


\section{Introduction}

Antimicrobial resistance (AMR) is an increasingly serious threat to global public health [1]. New resistance mechanisms are emerging and spreading globally, which reduces our means to treat common infectious diseases and therefore increasingly results in prolonged illness, disability, and death [1]. Current research suggests that the unexplored diversity of resistance mechanisms in environmental bacteria is a risk factor for the human population, and not only clinical pathogens that are equipped with AMR [2]. Natural environments are described as the origins and reservoirs of antimicrobial resistance genes (ARGs) [3]. Recent studies primarily focused on microbial communities and their ARGs in a wide range of human-influenced environments such as agricultural farmland, crop plants, food production systems, and wastewater treatment plants [4-7]. However, to fully understand the evolution, emergence and spread of antimicrobial resistance, it is crucial to also study natural systems that are not disturbed by anthropogenic factors.

Microbial diversity within natural microhabitats is an important bioindicator of changes in ecosystem function due to disturbances, such as exposure to pollutants, agricultural practices, climate change [8], and exposure to antimicrobials (reviewed in [9]). Generally, the effects of antimicrobials on single microorganisms or small consortia are well-known; however, understanding the consequences of antimicrobial exposure in complex, hostassociated microbiomes is a critical area where more research is required. It is important because the changes induced by antimicrobial exposure can have an immediate effect on host health [9]. Exposure to antimicrobial substances can severely impact microbial communities and often leads to selection and/or enrichment of ARGs $[10,11]$. A recent study by Mahnert et al. [12] demonstrated that loss of microbial diversity, due to cleaning in confined environments such as intensive care units and cleanroom facilities, correlates with an increase of antimicrobial resistance features. Despite this growing body of research that links antimicrobial exposure to changes in microbial communities, the community response to antimicrobial exposure in native environments is not yet understood [13].

Appropriate models for native microbial communities as well as for antimicrobial substances are required to obtain mechanistic insights into the effects of antimicrobial exposure on natural microbiota. Synthetic microbial communities are often used to simulate natural systems; however, they are less complex and less diverse and have lower functional connectivity than natural microbiota. Lichens form spatially limited microbial ecosystems consisting of a fungus (mycobiont), eukaryotic algae and/or cyanobacteria (photobiont), and thousands of different bacterial species [14-16]. Lichen-associated bacteria carry unique functional properties adapted to the holobiont, such as the production of antimicrobial substances and resistance towards toxic compounds $[14,17$, 18]. Although many lichens can persist under environmental extremes when they are dehydrated, they are generally vulnerable to slight changes in their microclimate [19], which substantially affects the fine-tuned symbiotic interplay [20]. We have selected lichens as promising systems for the exploration of complex, community-level responses of the microbiome to antimicrobial exposure due to their widespread use as models for classical symbioses as well as for bioindication/monitoring approaches [21]. Lichen thalli were exposed for a defined time period to representative clinical antimicrobials with narrow (colistin) and broadspectrum activity (tetracycline) [22, 23]. A bioactive alkylpyrazine was included to represent a novel, nonclinical antimicrobial [24] and glyphosate was included due to its wide but controversial use as herbicide with potential to affect the photobiont as well as non-target microorganisms $[25,26]$. Despite its toxicity, colistin is regarded as a last-line antimicrobial for the treatment of Gram-negative multi-resistant bacteria in many regions [27]. Our hypothesis was that all antimicrobials will reduce bacterial richness, suppress naturally dominant taxa, and induce (visible) dysbiosis in the lichen symbiosis. Furthermore, due to different target spectra and modes of action of the four selected antimicrobial compounds, we expected varying responses of the bacterial communities and enrichment of taxa with specific resistance features.

For this purpose, we studied bacterial community responses in the 'many-fruited pelt lichen' Peltigera polydactylon (Neck.) Hoffm. during exposure to a full therapeutic dosage and a gradient of sub-therapeutic dosages of four antimicrobials (colistin, tetracycline, glyphosate, alkylpyrazine) by DNA- and RNA-based amplicon sequencing along with a metagenomic dataset analyses. Specifically, we addressed the following questions: (i) Is there a specific microbial shift induced by antimicrobial exposure? (ii) Which taxa respond to antimicrobial exposure? And (iii) which genetic reservoir allows positive responders to thrive under antimicrobial exposure? Overall, this study provides key insights on how antimicrobial exposure shapes microbial communities in their natural environments and provides insights into the potential consequences of modern antimicrobial overuse.

\section{Materials and methods}

Collection of lichen material and antimicrobial treatments Peltigera polydactylon (Neck.) Hoffm. samples were collected in the proximate vicinity of a peri-urban area 
(Graz, Austria; $47^{\circ} 06^{\prime} 45.6^{\prime \prime} \mathrm{N}, 15^{\circ} 27^{\prime} 55.8^{\prime \prime}$ E). The healthy lichen population is part of a natural forest landscape with no industrial zones in the close proximity. It is located on an elevation and thus not affected by potential run-off from surrounding farmland. The sampling location represents a relatively pristine environment that will be affected by progressing urbanization. All samples were visually examined to detect and remove macroscopic contaminants, such as adhering moss and plant detritus, with sterile tweezers. Following the initial preprocessing steps, lichen samples ( $0.5 \mathrm{~g}$ dry weight) were placed into sterile Petri dishes.

Four different antimicrobials including colistin sulphate (Sigma-Aldrich, USA), tetracycline (Merck, Germany), glyphosate (commercial herbicide Roundup ${ }^{\circ}$ Alphée containing a glyphosate concentration of $7.20 \mathrm{~g} / \mathrm{l}$; Scotts Celaflor, Mainz, Germany), and an antimicrobial alkylpyrazine (5-isobutyl-2, 3-dimethylpyrazine 97\%, Sigma-Aldrich, USA) were used. As antimicrobial dosages could substantially impact the microbial community (reviewed in [8]), we selected dosages of the antimicrobials based on previous published studies that represented a full dosage (FD) and sub-therapeutic dosages (SD; 5-, 10-, and 20-fold dilution of FD) of each antimicrobial (in total 16 treatments; Table 1). Aqueous working solutions of each antimicrobial were prepared in sterile water as the solvent. Lichen samples were treated every $24 \mathrm{~h}$ with the antimicrobials over a period of 10 days by spraying the antimicrobial solutions (approximately $750 \mu \mathrm{L}$ per treatment) onto the surface of the lichens. Negative controls were implemented where lichens were sprayed with sterile water. The lichen samples were kept at room temperature. During the experiment, the average relative humidity range was between 58 and $65 \%$ (average $=61.8 \%$ ), whereas the average temperature was between 21 and $25^{\circ} \mathrm{C}$ (average $=23 \cdot 1^{\circ} \mathrm{C}$ ). Each treatment was performed in three biological replicates. After a 10-day incubation period, the samples were immediately transferred into a $15-\mathrm{ml}$ reaction tube with RNAlater stabilization solution (Ambion, Life Technologies, Germany) and stored at $-80^{\circ} \mathrm{C}$ until total nucleic acid extraction.

\section{Total nucleic acid extraction and CDNA synthesis}

Total deoxyribonucleic acid (DNA) and ribonucleic acid (RNA), from approximately $100 \mathrm{mg}$ of lichen sample, was extracted using the FastDNA ${ }^{\mathrm{Tm}}$ SPIN Kit for Soil (MP Biomedicals, Germany) and TRIzol ${ }^{\circ}$ Plus RNA
Purification Kit (Ambion, Life Technologies), respectively, following the manufacturer's instructions. To facilitate cell lysis, the samples were homogenized at room temperature using the FastPrep ${ }^{\text {Tx }}$ Lysing Matrix E and a FastPrep ${ }^{\oplus}$-24 Instrument (MP Biomedicals, Germany) for $3 \times 30 \mathrm{~s}$ at $6.0 \mathrm{~m} / \mathrm{s}$ with $1 \mathrm{~min}$ in-between cooling on ice. The RNA and DNA quality and quantity were examined by using the NanoDrop ${ }^{\text {Tw }}$ 2000/2000c Spectrophotometer and Qubit dsDNA BR and Qubit RNA HS Assay Kit (Thermofischer Scientific), respectively. To remove genomic DNA, total RNA (100 ng) was treated with DNase I (Epicentre; Lucigen, USA) and subsequently used to synthetize complementary DNA (cDNA) using 5X All-In-One RT MasterMix (Applied Biological Materials, Richmond, BC, Canada) according to the manufacturer's instructions. Prior further analysis, cDNA was diluted 10 times using nuclease-free water (Carl Roth, Germany).

\section{Quantification of bacteria in lichen samples}

Quantitative real-time PCR (qPCR) based on SYBR Green fluorescence was performed to quantify the total and active bacterial density after antimicrobial treatment using the primer pair 515f-927r [33, 34]. In total, 51 DNA and 51 cDNA samples were analysed (three biological replicates of each treatment and concentration). The $\mathrm{qPCR}$ reaction mix contained $1 \mu \mathrm{L}$ DNA/cDNA template, $5 \mu \mathrm{L}$ KAPA SYBR $^{\circ}$ FAST qPCR Master Mix (2X) (KAPA Biosystem, USA), $1 \mu \mathrm{L} 10 \mu \mathrm{M}$ of each primer, and $3 \mu \mathrm{L}$ ultrapure water. Fluorescence quantification was performed using the Rotor-Gene 6000 realtime rotary analyser (Corbett Research, Sydney, Australia) with initial denaturing at $95^{\circ} \mathrm{C}$ for $10 \mathrm{~min}$, followed by 40 cycles of denaturing at $95^{\circ} \mathrm{C}$ for $30 \mathrm{~s}$, annealing at $60^{\circ} \mathrm{C}$ or $62^{\circ} \mathrm{C}$ or $64^{\circ} \mathrm{C}$ for $30 \mathrm{~s}$, and extension at $72{ }^{\circ} \mathrm{C}$ for $30 \mathrm{~s}$ and a final melting curve. The Unibac-II fragment [33] was subjected to serial dilution (1:10) and run in two technical replicates to create qPCR standard. Negative and no-template controls were included in every run.

\section{Amplicon sequencing-based analyses of active and total bacterial communities}

Extracted DNA and cDNA were subjected for amplicon polymerase chain reaction (PCR) to target the bacterial

Table 1 Antimicrobial substances and their dosages used in this study

\begin{tabular}{llll}
\hline Antimicrobial & Full dosage & Sub-therapeutic dosages $(\boldsymbol{n}=\mathbf{3})$ & Reference \\
\hline Colistin* & $300 \mathrm{mg} / \mathrm{kg}$ & 60,30, and $15 \mathrm{mg} / \mathrm{kg}$ & [28, 29] \\
Tetracycline* & $1000 \mathrm{mg} / \mathrm{kg}$ & 200,100 , and $50 \mathrm{mg} / \mathrm{kg}$ & [30] \\
Glyphosate & $7.2 \mathrm{~g} / \mathrm{L}$ & $1.44,0.7$, and $0.36 \mathrm{~g} / \mathrm{L}$ & {$[31]$} \\
Alkylpyrazine & $0.66 \%$ & $0.13,0.07$, and $0.03 \%$ & {$[32]$} \\
\hline
\end{tabular}

*The required amount of these antimicrobials was calculated based on fresh weight of lichen thalli 
community. The primer set $515 \mathrm{f} / 926 \mathrm{r}$ was used to amplify the V4-V5 region of bacterial 16S rRNA gene [35]. The primers were constructed to contain an overhang at the $5^{\prime}$ end that was used to attach barcodes and Illumina flow cell adapter sequences in the subsequent PCR as previously described in the protocols of the Earth Microbiome Project [36]. Two technical replicates were performed for each sample. The quality of the PCR products was checked visually by loading to $1 \%$ agarose gel electrophoresis and using ultra-violet light with Biorad Universal Hood II Gel Doc System (Biorad, USA). Barcoded PCR products were pooled in equimolar concentrations after purification using Wizard $^{\circ}$ SV Gel and PCR Clean-Up kit (Promega). The pooled library was sent to the Genewiz (Leipzig, Germany) and sequenced using Illumina MiSeq (v2 reaction kit) $(2 \times 300$ bp paired-end).

\section{Bioinformatic analyses}

Due to low quality of the reverse reads, we only used forward reads for the amplicon sequencing analysis. To confirm robustness of the conducted data analysis, we compared forward and paired-end read datasets and observed a congruent result with both analysis strategies (Table S1 and Table S2). Due to a higher species richness that was observed in the dataset with forward reads (Fig. S1), we decided to exclusively use this dataset for further analyses. Bioinformatic analysis of the amplicon sequences was performed using the open-source QIIM E2 version 2018.4.0 pipeline (https://qiime2.org [37];). Demultiplex raw reads were imported to QIIME2 using 'qiime tools import'. Primer sequences were removed using the cutadapt plugin [38]. The DADA2 algorithm was used to quality filter and denoise demultiplexed sequences [39]. Subsequently, chimeric sequences were removed using the DADA2 chimera removal. The resulted amplicon sequences variants (ASVs) were taxonomically classified by using the VSEARCH classifier [40] against the reference database Silva v128 [41]. Prior further analysis, all reads assigned to Cyanobacteria and mitochondria were removed from the dataset.

The herein used metagenomic dataset (MG-RAST ID: mgm4551030.3) was previously reported [20] in the context of screening for arsenic-related functions. It was obtained from the same lichen population that was used for the present study. The raw data was re-analysed with updated bioinformatic tools to investigate ecological function and ARGs diversity in the lichen holobiont. Shotgun metagenomic reads were subjected to adapter trimming and quality filtering using Trimmomatic and VSEARCH $[40,42]$. The filtered reads were used as input files for taxonomic profiling using Kaiju [43] and for assembly using metaSPAdes with default parameters [44]. The filtered reads were mapped back to the assembled contigs using Bowtie2 [45]. The assembled contigs were annotated using the blastx algorithm in DIAMOND [46] against eggNOG version 4.5 database [47] and the manually curated antimicrobial resistance gene database (deepARG) [48] to perform ecological function and antimicrobial resistance genes profiling. To minimize the risk of false positives, reads were defined as ARG-like reads at the cut-off $E$ value of $10^{-10}$ and similarity of $35 \%$ as previously described by $[49,50]$. FeatureCounts [51] were used to align metagenomic reads to the annotated contigs and to obtain total read numbers, respectively. Amplicon sequences were deposited at the European Nucleotide Archive (ENA) under the project number PRJEB37912.

\section{Statistical analyses}

The $\mathrm{R}$ version 1.2 (R Core Team, 2017) was used to perform general statistical analysis and visualize results. Significant differences $(P<0.05)$ of bacterial gene copy numbers were analysed using the non-parametric Kruskall-Wallis test. The ASV tables and taxonomic classifications that were generated with QIIME2 were imported into $\mathrm{R}$ via phyloseq [52]. The number of sequences from each amplicon sequencing library was normalized to the lowest number of read counts (1009 reads per sample) by randomly selecting subsets of sequences. A taxonomy summary of the top 100 most abundant ASV at class level was visualized by using the integrated bar plots. Differences in microbial alpha diversity based on the number of identified ASVs and the Shannon index were analysed using the non-parametric Kruskall-Wallis test followed by the paired difference test, Wilcoxon signed-rank test. The beta diversity assessment based on normalized Bray-Curtis dissimilarity matrix was subjected to the Adonis test (999 permutations) to determine the effect of antimicrobial exposure and different dosages on microbial community structures. The distance matrices were visualized using non-metric multidimensional scaling (NMDS) plots. The analyses mentioned above were performed using the $R$ package vegan [53]. We also correlated total and active bacterial community matrix distance through partial Mantel tests (corrected for spatial distance) with 999 permutations. Bacterial genera associated with each antibiotic treatment were identified by LefSe (liner discriminant analysis effect size) as implemented in MicrobiomeAnalyst [54-56]. The threshold for the linear discriminant analysis (LDA) was set to 2 with a $P$ value cut-off of 0.05 . Finally, the correlation analysis implemented in the ggpubr package [57] was used to calculate Spearman coefficients for correlations between bacterial genera and different antimicrobial compounds. 
Complementary quantification of the $m c r 1$ gene by qPCR To quantify AMR activation of the positive responders at mRNA level, we performed a targeted qPCR analysis of the $m c r 1$ gene. The $m c r 1$ gene was selected, because it is the only known gene that confers colistin resistance. We therefore expected that it is present in lichenassociated bacteria that thrive under colistin exposure. The qPCR experiments were conducted using the primer pair mcr1FP-mcr1RP as previously described [58]. A mcr 1 standard was obtained from ten-fold serial dilutions of the genomic DNA of a colistin-resistant Escherichia coli isolate. The isolate is part of the culture collection of the Department of Internal Medicine, Medical University, of Graz. Extracted cDNA from colistintreated and control samples was subjected to qPCR analyses using the Rotor-Gene 6000 real-time rotary analyser (Corbett Research) with previously described parameters [58].

\section{Results}

\section{The Peltigera microbiome and antimicrobial resistance} genes

A total of $1.67 \times 10^{6}\left(1.63 \times 10^{4}\right.$ per sample mean $)$ highquality reads were obtained in the amplicon sequencing approach. From the metagenomic dataset, a total of 13.9 $\times 10^{6}$ reads were annotated using the eggNOG database, while a total of $3.04 \times 10^{5}$ reads $(0.82 \%$ of total reads) was assigned to ARGs using the deepARG database. According to the Kaiju classifier, we detected metagenomic contigs that were classified as Cyanobacteria. Amplicon libraries were also dominated by Nostocaceae (Cyanobacteria phylum) sequences. These contigs and the respective raw sequences were not evaluated as part of the bacterial community because Nostoc represent the wellstudied, homogenous phototobiont in Peltigera, and they commonly carry only a few distinct ARGs, for example mtrA (multidrug resistance gene). After filtering nontarget taxa, a total of $8.6 \times 10^{5}\left(8.4 \times 10^{3}\right.$ per sample mean) amplicon sequencing reads were retained and assigned to 3124 bacterial ASVs. Comparison between community assessments on metagenome, DNA amplicon, and RNA amplicon level revealed that the general bacterial community structure showed a certain congruent at class and order level across the dataset regardless of different approaches (Fig. 1a-c). Alphaproteobacteria were the most dominant class in the Peltigera-associated microbiome in the non-treated samples with a relative abundance (RA) of 29-43\%. The other predominant classes were Bacteroidetes (17-28\%), and Gammaproteobacteria/Betaproteobacteria (9-17\%). Taxonomic analysis revealed four highly abundant orders, i.e., Rhizobiales (17.6\%, average RA from metagenomic and amplicon sequencing dataset), Sphingobacteriales
(12.3\%), Sphingomodales (10.3\%), and Betaproteobacteriales (8.7\%).

We conducted a general functional analysis, which focused on functions that could directly affect the symbiosis. In the overall dataset, the majority of metagenomic reads (65\%) was assigned to bacterial proteins. Therein, we detected numerous reads $(1.4 \%)$ assigned to Ton and Tol transport systems which are involved in iron uptake. Many of these reads were derived from Sphingomonas, Methylobacterium, and Mucilaginibacter. Genes that are involved in vitamin production such as cobalamin biosynthesis protein and folate metabolism $(0.3 \%)$ were also detected within contigs derived from these taxa. In addition, bacterial porin proteins such as carbohydrateselective porin and aquaporin that may be involved in carbohydrate metabolism and drought stress were also detected $(0.03 \%)$. Using the eggNOG database, we found that a high number of bacterial reads (2.6\%) were assigned to defence mechanism function. The majority of those proteins $(25.5 \%)$ were annotated as part of an ABC-transport system.

More specific profiling of antimicrobial resistance genes in the metagenomic dataset against the deepARG database resulted in the detection of 728 ARGs spanning 30 antimicrobial classes (Fig. 1d). Most of the identified ARGs originated from Proteobacteria (28\% of Alphaproteobacteria and $20 \%$ of Beta/Gammaproteobacteria, Fig. S2). A total of $80.5 \%$ detected ARGs were classified to macrolide-lincosamide-stretogramin (MLS) multidrug classes, bacitracin, beta lactam, and polymyxin. This finding indicated a high diversity, but a low abundance, of ARGs embedded in the lichen metagenome (Fig. 1d).

\section{Responses to exposure to antimicrobials at phenotype and genotype level (richness and diversity)}

Antimicrobial treatments resulted in phenotypic changes to the exposed Peltigera thalli. A change of colour from drab grey-green to dark brown in lichen samples treated with the full dosage of the alkylpyrazine was observed after 3 days of continuous exposure in comparison to the control (Fig. S3). A similar phenotypic change was also observed in samples treated with the full dosage of glyphosate after 5 days, which became more obvious after 8 days of continuous exposure. We did not observe a change of colour in lichen thalli that were treated with sub-therapeutic dosages of alkylpyrazine and glyphosate as well as colistin and tetracycline treated samples in comparison to the control during the whole experiment. These changes indicate that the naturally occurring cyanobacteria were negatively affected by the alkylpyrazine and glyphosate treatments due to algicidal properties of these antimicrobials.

Different exposures to antimicrobials and their respective dosages affected bacterial richness $(P<0.001, P$ 


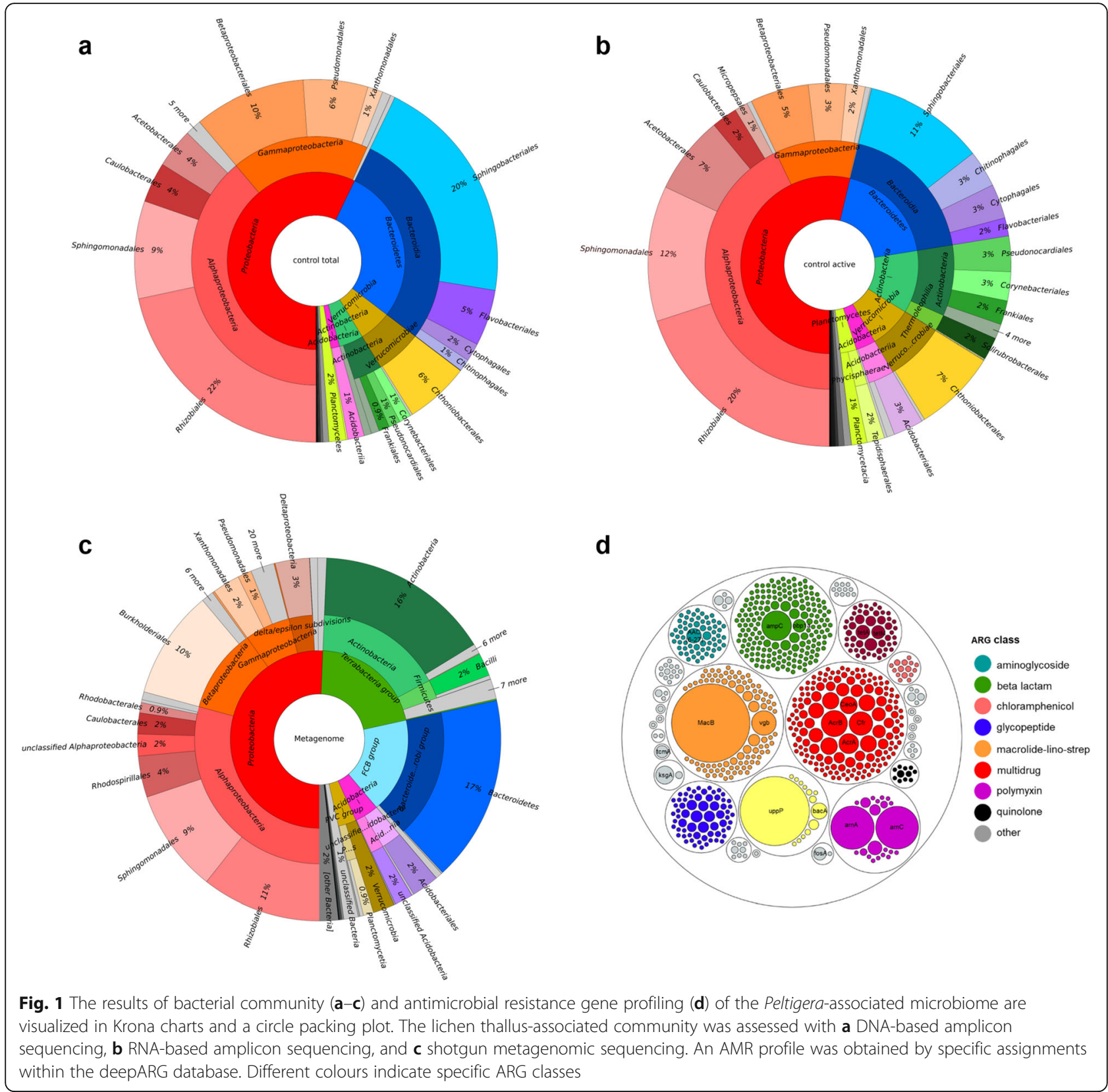

$=0.010$, respectively, Table S3) according to the Shannon diversity index $(H)$, whereas the assessment type (DNA- or RNA-based amplicons) did not have any effect on the alpha diversity $(P=0.632$, Table S3). When analysed separately, each of the employed dosages of alkylpyrazine and glyphosate showed no significant changes in bacterial richness when compared to the untreated control group ( $P>0.05$, Table S3). In contrast, highly significant changes were observed for the colistin and tetracycline treatments $(P<0.001$, Table S3). Increased dosage of these antimicrobial substances resulted in substantially reduced bacterial richness. The highest impact was observed in the samples exposed to the full dosages of colistin $\left(H^{\prime}=1.7\right.$ and $H^{\prime}=1.7$-total and active bacteria, Table S4) and tetracycline $\left(H^{\prime}=1.4\right.$ and $\left.H^{\prime}=2.5\right)$ in comparison to non-treated samples $\left(H^{\prime}=4.3\right.$ and $H^{\prime}=$ 4.3).

To investigate the impact of the antimicrobial exposure on the bacterial community structure, beta diversity analysis was performed using Bray-Curtis matrix distance in combination with Adonis and visualized using a non-metric multidimensional scaling (NMDS) plot. The antimicrobial type was found to be the main driver of the bacterial community structure $\left(R^{2}=0.265, P=\right.$ 0.001 , Table 2, Fig. 2a), whereas the other factors, such as antimicrobial dosage or type of community (total or 
active bacterial fraction) only explained a small amount of the variation $\left(P=0.001 ; R^{2}=0.076\right.$ and $R^{2}=0.043$, respectively). A complementary Mantel test showed a highly significant correlation of both, the total bacterial community and the active community $(P=0.001, R=0.719)$. This indicated a high congruent between these approaches. When the data was separated according to the antimicrobial substance, we observed that antimicrobial dosage in each dataset had a substantial impact on bacterial community structure $(P=$ $\left.0.001, R^{2}=0.255-0.571\right)$. In ordination space, a clear clustering was observed between treated and non-treated samples where samples that were treated with higher dosage are further apart from non-treated samples (Fig. 2b-e). A noteworthy result was that a higher impact of antimicrobial dosage was observed in the colistin dataset $\left(R^{2}=0.571\right)$ compared to other datasets.

\section{Antimicrobial exposure induces changes in bacterial community composition without altering bacterial abundances}

To investigate the total and active bacterial abundance of the lichen-associated bacteria after antimicrobial

Table 2 Effect of antimicrobial treatment, dosages, and sample type (DNA or RNA) on bacterial community structure ( $\beta$-diversity). A complementary statistical analysis was conducted in order to identify factors with a significant effect on the bacterial community

\begin{tabular}{|c|c|c|}
\hline \multirow[t]{2}{*}{ Factor } & \multicolumn{2}{|c|}{ Microbial community similarities } \\
\hline & $R^{2}$ value & $P$ value \\
\hline \multicolumn{3}{|l|}{ All datasets } \\
\hline Antimicrobials & 0.265 & $0.001^{*}$ \\
\hline Dosage & 0.076 & $0.001^{*}$ \\
\hline Type & 0.043 & $0.001^{*}$ \\
\hline \multicolumn{3}{|l|}{ Colistin dataset } \\
\hline Dosage (D) & 0.528 & $0.001^{*}$ \\
\hline Type (T) & 0.069 & $0.001^{*}$ \\
\hline $\mathrm{D} \times \mathrm{T}$ & 0.131 & $0.001^{*}$ \\
\hline \multicolumn{3}{|c|}{ Tetracycline dataset } \\
\hline Dosage (D) & 0.471 & $0.001^{*}$ \\
\hline Type (T) & 0.065 & $0.007^{*}$ \\
\hline $\mathrm{D} \times \mathrm{T}$ & 0.097 & 0.087 \\
\hline \multicolumn{3}{|c|}{ Alkylpyrazine dataset } \\
\hline Dosage (D) & 0.340 & $0.001^{*}$ \\
\hline Type (T) & 0.134 & $0.001^{*}$ \\
\hline $\mathrm{D} \times \mathrm{T}$ & 0.128 & $0.011^{*}$ \\
\hline \multicolumn{3}{|c|}{ Glyphosate dataset } \\
\hline Dosage (D) & 0.255 & $0.001^{*}$ \\
\hline Type (T) & 0.070 & $0.001^{*}$ \\
\hline $\mathrm{D} \times \mathrm{T}$ & 0.099 & 0.769 \\
\hline
\end{tabular}

*Significant differences $(P \leq 0.05)$ were assessed with the Adonis test exposure, a quantitative polymerase chain reaction (qPCR) approach with specific bacterial primers, targeting the 16S ribosomal RNA gene was performed. Total bacterial rRNA gene abundance ranged between $1.02 \times$ $10^{7}$ and $1.90 \times 10^{8} 16 \mathrm{~S}$ rRNA gene copies per ng extracted DNA whereas active bacterial rRNA gene abundance ranged between $4.40 \times 10^{3}$ and $6.35 \times 10^{4} 16 \mathrm{~S}$ rRNA gene copies per ng extracted RNA (Table S5). Overall, statistical significance tested using the KruskalWallis test showed no effect of antimicrobial treatment on bacterial abundance $(P>0.05)$.

In order to visualize taxonomic composition of the lichen holobiont after antimicrobial exposures, bar plots showing the 100 most abundant bacterial ASVs were constructed (Fig. 3). Distinct taxonomical changes were observed after antimicrobial exposure depending on the type of antimicrobial and their dosages. Taxonomical shifts after exposure to colistin were more similar to tetracycline exposure whereas glyphosate exposure showed a more similar taxonomical shift to alkylpyrazine exposure.

Most remarkably, the abundances of Pseudomonadaceae family (Gammaproteobacteria), increased in response to colistin exposure. The relative abundance of Pseudomonadaceae gradually increased in response to colistin exposure which reached up to $79.6 \%$ and $80.3 \%$ in the total and active bacterial fraction, respectively, when exposed to the full dosage of colistin. In contrast, this taxon represented only 5.9 and $3.3 \%$ (total and active bacterial fraction, respectively) in non-treated samples. A similar pattern was observed after exposure to tetracycline where the relative abundance increased up to $49.4 \%$ and $31.1 \%$ in the treatment with the full dosage. In contrast, the relative abundances of Sphingobacteriaceae as well as Sphingomonadaceae decreased to below $0.2 \%$ and $0.6 \%$ after full dosage exposure of these antimicrobial substances. Taxonomical shifts in the bacterial families were also observed after alkylpyrazine exposure with an increase in Beijerinckiaceae, ranging from $26.5 \%$ and $20.8 \%$ (total and active bacterial fraction, respectively), with the lowest concentration of alkylpyrazine, to $30.4 \%$ and $31.1 \%$ in the full dosage, in comparison to non-treated samples (17.3\% and $16.4 \%)$. Overall, exposure to antimicrobial substances at different dosages induced shifts in the bacterial community structure (Fig. 3a, b). Moreover, an indication of microbial imbalance due to selectively enriched low abundant taxa was observed in samples treated with colistin and tetracycline.

\section{Putative roles of the identified bacterial responders}

Calculation of linear discriminant analysis effect size (LEfSe) was performed to identify taxa that were significantly affected by antimicrobial treatments. The analysis indicated that 15 and 12 bacterial genera were affected 

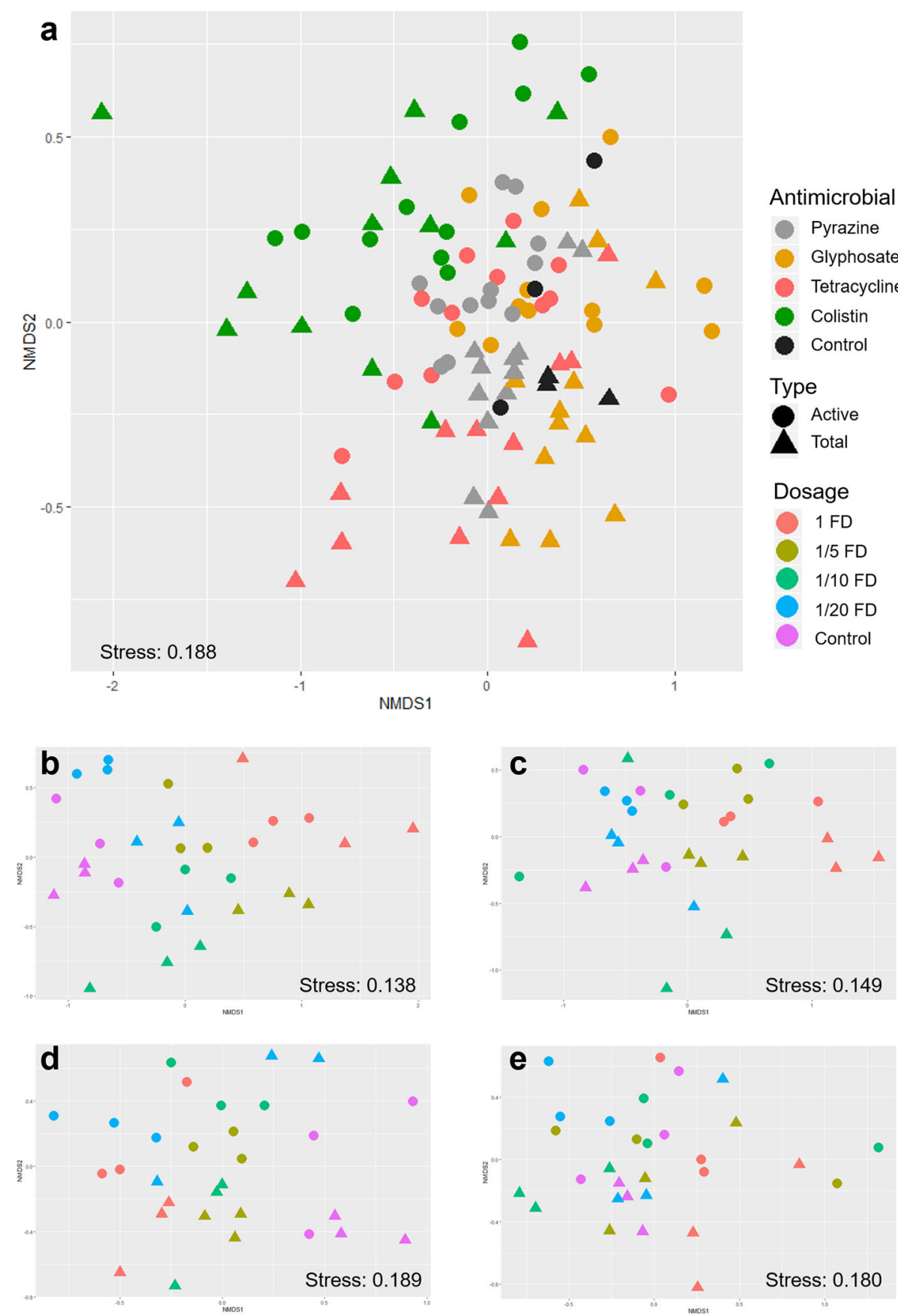

Fig. 2 Community clustering of bacterial composition (total and active bacterial community) in lichens treated with different antimicrobial compounds is visualized in two-dimensional Bray-Curtis NMDS plots. The plots are based on $\mathbf{a}$ all datasets, $\mathbf{b}$ colistin dataset, $\mathbf{c}$ tetracycline dataset, $\mathbf{d}$ glyphosate dataset, and $\mathbf{e}$ alkylpyrazine dataset

in the total and active bacterial community of antimicrobial-treated and non-treated samples, respectively (Fig. S4). Pseudomonas and Curtobacterium were consistently enriched under colistin exposure and Burkholderia was consistently enriched under tetracycline exposure (Fig. S4). The analysis also indicated that Methylobacterium, Acidiphilium, and an unidentified member of Beijerinckiaceae were consistently enriched under alkylpyrazine exposure. In complementary analyses, we statistically examined correlations between the relative abundance of bacterial genera and antimicrobial concentrations. To minimize spurious correlation, we selected only bacterial genera with a relative abundance above $2 \%$ in the whole dataset. Acidiphilium, 


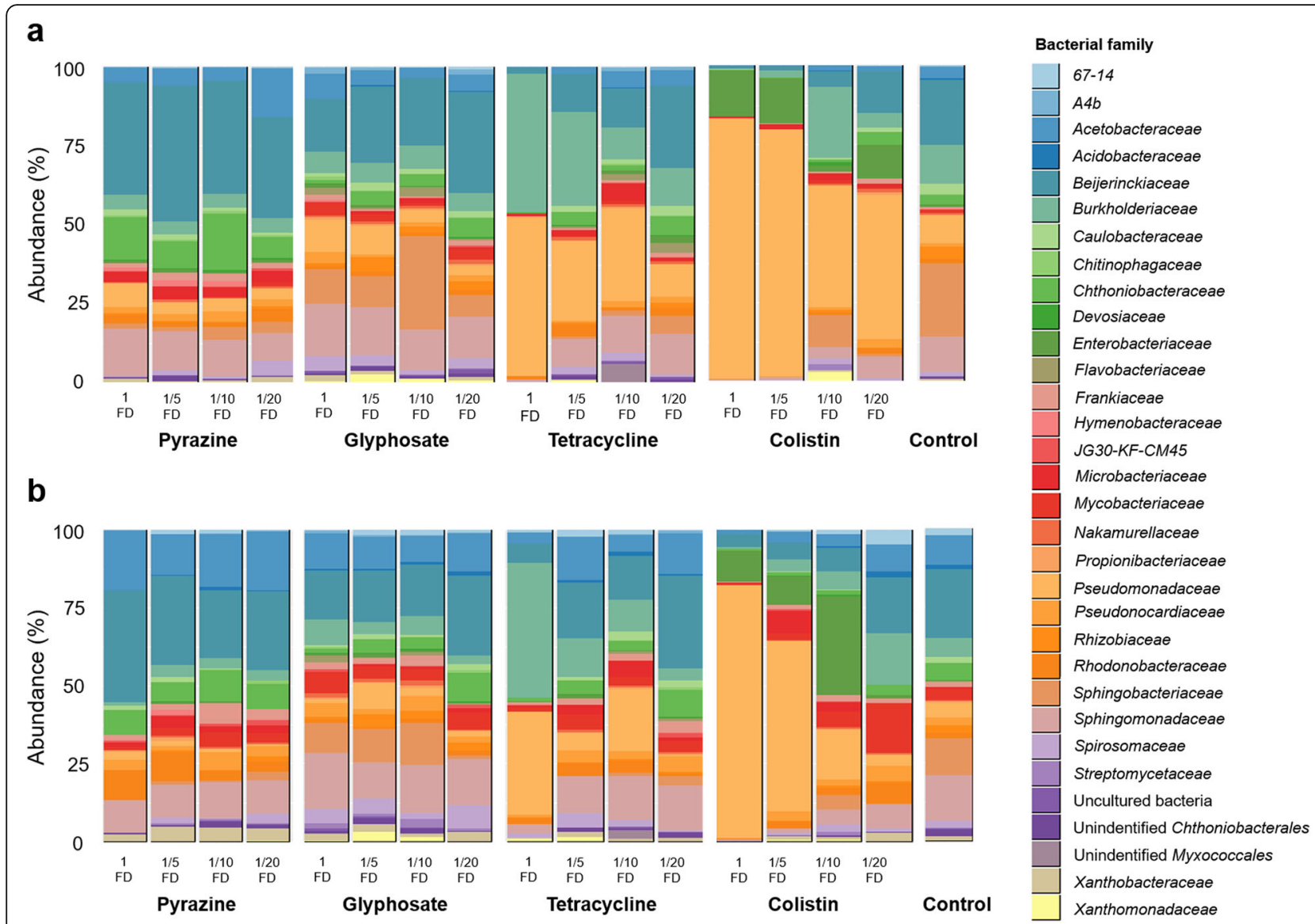

Fig. 3 Relative abundance plot of the top 100 most abundant bacterial families in lichen samples with or without antimicrobial treatment. A differential assessment of the $\mathbf{a}$ total bacterial community and $\mathbf{b}$ active bacterial community was conducted. For each included treatment, antimicrobials at their full dosage as well as three sub-therapeutic dosages were applied

Burkholderia, and Sphingomonas were found to consistently negatively correlate to increase dosage of colistin in both, total and active bacterial community. The latter taxon was also negatively correlated with increasing tetracycline dosage. Several genera, i.e., Acidiphilium, Flavobacterium, Mucilaginibacter, Methylobacterium, and Rhizobium were identified to also negatively correlate to increased dosages of tetracycline in the total bacterial community dataset. We further identified bacterial genera that were positively correlated to increased antimicrobial dosage in both, the total and the active bacterial community $(P<0.05$, Fig. $4 \mathrm{a}, \mathrm{b})$. The relative abundance of Pseudomonas was found to correlate to increased dosage of colistin and tetracycline in the total and active bacterial community. Burkholderia was found to correlate to an increased dosage of tetracycline while Sphingomonas was correlated to an increased dosage of glyphosate (Fig. 4a, b).

To address the question why distinct responders could thrive under pressure caused by specific antimicrobials, we investigated contigs that were assigned to each of the responders from the metagenome dataset and compared the presence/absence of specific antimicrobial resistance genes. From the detected ARGs in the Peltigera metagenome, a network of co-occurring ARGs was constructed to visualize shared and unique ARGs of the selected responders (Fig. 4c). A high proportion of multidrug resistance and quinolone were shared between the responders. Sphingomonas $(n=102)$, Pseudomonas $(n=83)$, and Methylobacterium $(n=69)$ had a higher number of multidrug resistance genes in comparison to other responders such as Burkholderia $(n=47)$ and Rhizobium $(n=51)$. Moreover, a higher number of multidrug resistance genes in Sphingomonas and Methylobacterium contigs may explain how responders could thrive during exposure to non-clinical antimicrobial substances, glyphosate and alkylpyrazine, respectively. Despite the high occurrence of shared ARG genes, mcrl, a colistin resistance gene, was detected in Pseudomonas-derived contigs indicating that this gene may be responsible for its thriving under colistin exposure. When copy numbers of the morl transcript were 


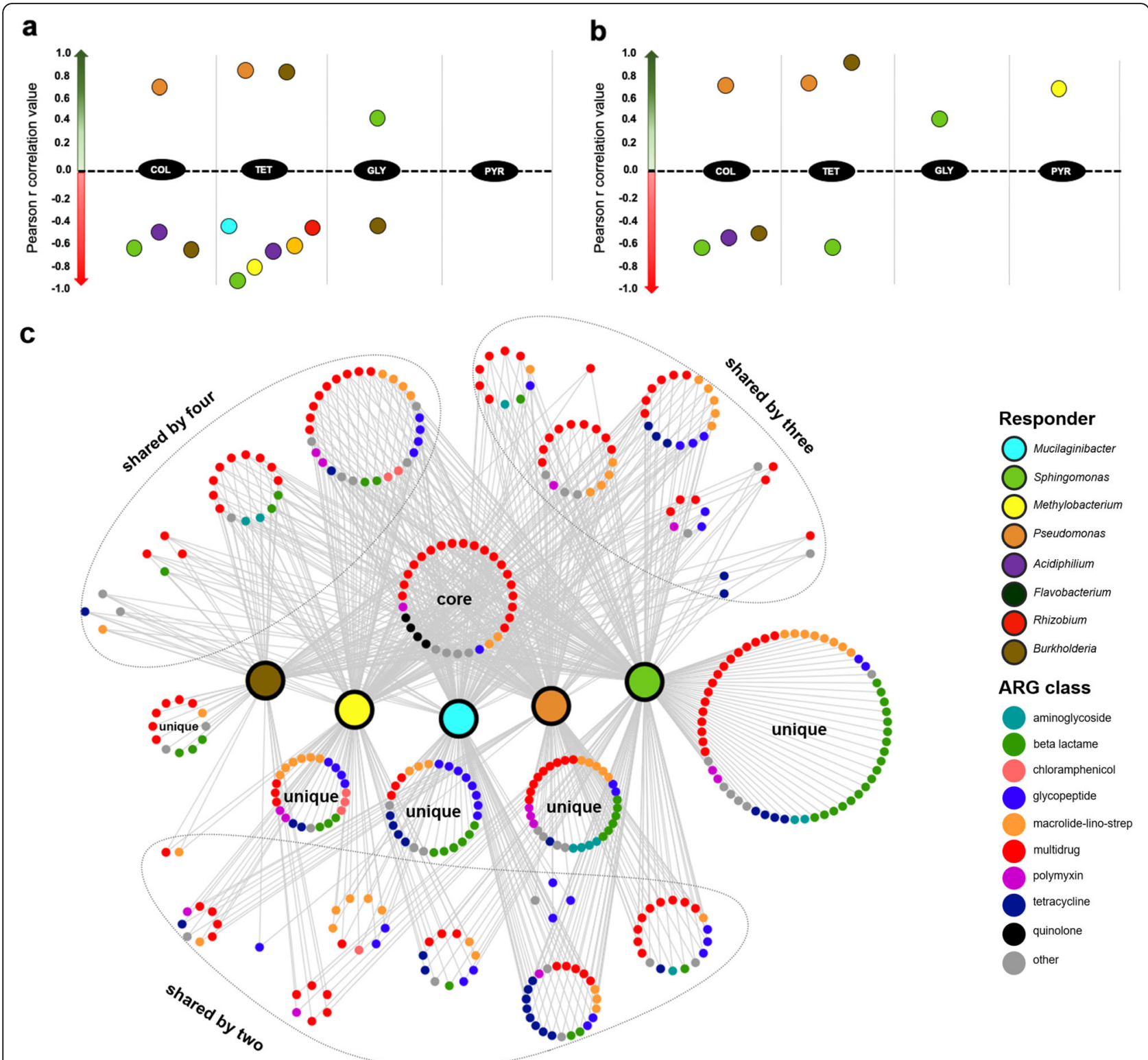

Fig. 4 Correlation analysis between relative abundances of bacterial genera and antimicrobial dosages $(\mathbf{a}, \mathbf{b})$ and network visualization of shared and unique antimicrobial resistance genes (ARGs) from selected bacterial responders (c). Responders (indicated with different colours) are shown in the $\mathbf{a}$ total bacterial community and $\mathbf{b}$ active bacterial community. Only correlations with $P<0.05$ are displayed. The nodes in the network are coloured according to ARG classes. COL, colistin; TET, tetracycline; GLY, glyphosate; PYR, 5-isobutyl-2,3-dimethylpyrazine

analysed via $\mathrm{qPCR}$ in colistin-treated and non-treated samples, significantly $(P=0.007)$ higher transcript numbers were found in colistin-treated samples irrespective of the dosage in comparison to non-treated samples (Table S6).

All positive and negative responders were shown to carry tetracycline resistance genes. A higher number of tetracycline resistance genes was found in negative responders Mucilaginibacter $(n=19)$ and Sphingomonas $(n=22)$ derived contigs in comparison to the positive responders, i.e., Burkholderia $(n=3)$ and Pseudomonas $(n=4)$. We also detected tet $A B(46)$ and tet $A B(60)$ that was only shared between Mucilaginibacter and Sphingomonas. These genes encode $\mathrm{ABC}$ transporters that confer resistance to tetracycline.

\section{Discussion}

Our data highlights that the natural microbiome of Peltigera comprises highly diverse and low abundant intrinsic ARGs, which provide a retrievable basis to cope with antimicrobial pressure. Similar to other relatively pristine environments, ARGs were found to be ubiquitous and to 
harbour a high number of different efflux pump systems $[13,59,60]$. In nature, ARGs fulfil various roles and are commonly involved in processes such as detoxification and molecular signalling. However, the same mechanisms can also serve as an essential feature of nosocomial pathogens to overcome high (toxic) antimicrobial concentrations that are found in clinical settings [59, 61]. The high diversity of ARGs that was detected in the present study, reflects the natural complexity of microbial communities that are commonly associated with lichens, and have previously been shown to provide metabolic versatility that facilitates plasticity of the lichen holobiont [13, 62].

Our study provides the first detailed insights into community-level response to antimicrobial exposure in a pristine system. In agreement to previous reports and, as expected, a higher impact of antimicrobial exposure was observed on the bacterial community structure compared to the bacterial abundance [26, 30]. We observed a specific shift in the taxonomic composition and community structure of native bacteria as a response to antimicrobial exposure. Despite minor variations, comparable and congruent alpha and beta diversity results between DNA- and RNA-based amplicon sequencing indicated that both of the approaches reflected how antimicrobial exposure shaped the bacterial community. Both approaches led to the identification of mostly overlapping responder taxa. Moreover, the similarity between the DNA and RNA approach indicates that the majority of bacteria in lichen was active. We observed a relatively lower effect after exposure to non-clinical antimicrobial compounds, i.e., glyphosate and alkylpyrazine despite their broad activity spectrum compared to the clinical antimicrobial compounds, i.e., colistin and tetracycline. This indicated that the bacterial community is more resilient towards non-clinical antimicrobials. We hypothesize that the diversity of unspecific multidrug efflux pumps that are shared among Peltigera-associated bacteria may play a major role in the observed resilience. Distinct taxa such as Sphingomonas and Methylobacterium are equipped with a high number of multidrug resistance features, which are likely important for their resilience towards non-clinical antimicrobial compounds. Multidrug efflux pumps, especially $\mathrm{ABC}$ transporters, are known to contribute to herbicide resistance [63, 64]. Despite this, there are no studies reporting resistance genes against pyrazines, producers of these compounds can be frequently found in nature and more specifically in the microbiota of other lichens $[65,66]$. Thus, Peltigera-associated bacteria may likely encounter these antimicrobial compounds in nature whereby multidrug efflux pumps likely provide the best means for detoxification [61].

Certain taxa in the lichen microbiome that naturally occur in low abundances but are equipped with specific resistance features, increased in response to colistin and tetracycline. Antimicrobial exposure might have created a temporary 'biological vacuum' as a result of the reduction of bacterial diversity and therefore created a new niche for more resilient bacteria (responders) for recolonization and bloom [67, 68]. Pseudomonas consistently increased in response to colistin and tetracycline. The morl gene, which encodes a phosphoethanolamine transferase, was found among Pseudomonas-assigned contigs in the metagenomic dataset and provides an explanation for the resilience against colistin. The $\mathrm{mcr} 1$ transcripts were also higher in colistin-treated samples in comparison to non-treated samples when assessed with a complementary qPCR approach. This gene constitutes the only known mechanism to confer colistin resistance by altering antimicrobial-specific binding sites (reviewed in [69-71]). It remains unclear how the positive responders, i.e., Pseudomonas and Burkholderia could thrive under tetracycline exposure, because negative responders were shown to also harbour tetracycline resistance genes. Nevertheless, it is worth to mention that the presence of genes in a metagenomic library do not necessarily imply their functional expression [72]. This is important in the context of our study since we detected a high number of tetracycline resistance genes in Sphingomonas and Mucilaginibacter despite the observed negative effect of tetracycline exposure on their relative abundance. Nevertheless, other factors such as increased bacterial resilience through biofilm formation, the host response and nutrient availability that were not assessed in this study, might be involved in increased abundance of distinct taxa under specific antimicrobial exposure [73, 74]. Therefore, further studies based on metatranscriptomic and metaproteomic approaches will be needed to identify genes, proteins, and pathways that are associated with bacterial community responses during antimicrobial exposure.

We showed that naturally dominant taxa, such as Sphingomonas and Mucilaginibacter, were negatively correlated to increased concentrations of clinical antimicrobials. Lichens are known to harbour bacteria with functional guilds that play an essential role as probiotics and detoxifiers [18]. Dominant taxa, such as Sphingomonas and Mucilaginibacter that encoded for various transport machineries, such as Ton- and Tol-dependent transport as well as porins, are suggested to play important roles in iron metabolism and transport as part of a survival strategy in the lichen holobiont $[14,75]$. Following antimicrobial exposure, it was observed that the native microbiota may be restored to the initial composition; however, the restoration remains often incomplete (reviewed in [76]). Therefore, collateral damage of dominant and crucial taxa from prolonged exposure to antimicrobials may disrupt the fine-tuned 
symbiotic interplay in lichens even if they harbour resistant taxa. In the present study, we also observed a bloom of low abundant taxa that carry features that are known to confer colistin resistance. This is relevant in the context of potential spread of natural AMRs to clinical settings, which currently rely on this antibiotic. Lichens that cover up to $8 \%$ of the total terrestrial surface [77] may be increasingly affected by anthropogenic activities in the future such as overuse of antimicrobial substances [78]; the use of antimicrobials for agricultural purposes is predicted to increase at least $99 \%$ by 2030 [79]. Increased antimicrobial pressure in natural AMR reservoirs may increase the risk for resistance transmission to opportunistic human pathogens $[59,80]$.

\section{Conclusion}

Lichens are ideal model organisms to mechanistically study how antimicrobial exposure affects native microbiota due to their well-defined, highly diverse bacterial colonizers. All antimicrobial substances showed an impact on the microbiome and we identified distinct positive as well as negative responders. Microbial dysbiosis caused by exogenic antimicrobials can result in a bloom of naturally low abundant taxa (positive responders) with specific AMR features. Bacteria assigned to the genera Pseudomonas, Sphingomonas, Burkholderia, and Methylobacterium were identified as positive responders; many species of these genera are already well-known nosocomial pathogens in clinical environments. The findings of the present study indicate that in situ exposure of microbial communities can facilitate the identification of AMR-carriers with resistance features of opportunistic human pathogens and is thus a valuable tool to explore their emergence. Moreover, these and future findings may be translatable into new management strategies for AMR-affected environments, e.g., alternating use of different antimicrobials in clinical settings to reduce specific antimicrobial pressure that was shown to result in a bloom of distinct resistance carriers.

\section{Supplementary Information}

The online version contains supplementary material available at https:/doi. org/10.1186/s40168-020-00982-y.

Additional file 1. Table S1. Comparison of the alpha diversity in forward and paired-end read datasets. Table S2. Comparison of the beta diversity in forward and paired-end read datasets. Table S3. Effect of antimicrobial treatment, dosages, and sample type (DNA or RNA) on lichen associated bacterial richness (alpha diversity) according to the Shannon diversity index. Table S4. Lichen-associated bacterial richness (alpha diversity) according to the Shannon diversity index following exposure to antimicrobial compounds. Table S5. Real time qPCR-based assessment of total bacterial $16 \mathrm{~S}$ rRNA gene copy numbers in lichens after exposure to different antimicrobial substances. Table S6. Real time qPCR-based assessment of $\mathrm{mcr}-1$ resistance gene copy numbers in lichens after exposure to colistin. Figure S1. Rarefaction curves showing the number of ASVs that were observed in lichens treated with different antimicrobial compounds.
Rarefaction curves are based on (a-e) the forward-read-only dataset and $(f-j)$ the paired-end read dataset and derived from the $(a, f)$ colistin $(b, g)$, tetracycline $(c, h)$, alkylpyrazine $(d, i)$ and glyphosate $(e, j)$ treatments. Figure S2. Identification of carriers of the detected antimicrobial resistance genes. The annotation was conducted using the metagenome classifier Kaiju and visualized with the integrated bubble plot tool. Figure S3. Phenotypes of lichen samples that were treated with full dosages of antimicrobial compounds i.e. colistin, tetracycline, glyphosate, and alkylpyrazine in comparison to the untreated control. Representative lichen samples were documented on day 3,5 and 8 after the first spray application of the antimicrobial substances. Figure S4. Identification of responders to different antimicrobial compounds by LEfSe (Linear discriminant analysis effect size). The analyses are based on (a) the total and (b) the active bacterial community. Only bacterial genera with a LDA score above 2 and cut-off $P$ values below 0.05 were included.

\section{Abbreviations}

AMR: Antimicrobial resistance; ARG: Antimicrobial resistance gene; FD: Full dosage; DNA: Deoxyribonucleic acid; RNA: Ribonucleic acid; CDNA: Complementary DNA; PCR: Polymerase chain reaction; qPCR: Realtime (quantitative) polymerase chain reaction; ASV: Amplicon sequences variant; NMDS: Non-metric multidimensional scaling; MLS: Macrolidelincosamide-stretogramin

\section{Acknowledgements}

The authors gratefully thank Barbara Fetz (Graz) for her support during DNA extractions from lichen thalli. The authors would like to thank Dr. Kathryn

Walker (University Canterbury) for English revision and discussion.

\section{Authors' contributions}

GB, TC, MG, and WAW conceived and designed the study. WAW, SE, and TRG performed the laboratory work. WAW and PK performed bioinformatic analysis. WAW, PK, TC, and GB wrote the manuscript. All authors read and approved the final version of the manuscript.

\section{Funding}

This study was supported by a grant from the FWF (Austrian Science Fund) and the federal state government of Styria to G.B. (P29285-BBL).

\section{Availability of data and materials}

Raw sequencing data for each sample used in this study was deposited at the European Nucleotide Archive (ENA) in the FASTQ format and is available under the Bioproject accession number PRJEB37912.

Ethics approval and consent to participate

Not applicable.

\section{Consent for publication}

Not applicable.

Competing interests

The authors declare that they have no competing interests.

\section{Author details}

${ }^{1}$ Institute of Environmental Biotechnology, Graz University of Technology, Graz, Austria. ${ }^{2}$ Institute of Biology, University of Graz, Graz, Austria.

Received: 29 April 2020 Accepted: 16 December 2020

Published online: 27 January 2021

References

1. World Health Organization. Antimicrobial resistance. 2018. https://www.who. int/news-room/fact-sheets/detail/antimicrobial-resistance. Accessed $20 \mathrm{Apr}$ 2020 .

2. von Wintersdorff CJ, Penders J, van Niekerk JM, Mills ND, Majumder S, van Alphen LB, et al. Dissemination of antimicrobial resistance in microbial ecosystems through horizontal gene transfer. Front Microbiol. 2016;7:173.

3. Martínez JL. Natural antibiotic resistance and contamination by antibiotic resistance determinants: the two ages in the evolution of resistance to antimicrobials. Front Microbiol. 2012;3:1. 
4. Cacace D, Fatta-Kassinos D, Manaia CM, Cytryn E, Kreuzinger N, Rizzo L, et al. Antibiotic resistance genes in treated wastewater and in the receiving water bodies: a pan-European survey of urban settings. Water Res. 2019;162: 320-30.

5. Cernava T, Erlacher A, Soh J, Sensen CW, Grube M, Berg G. Enterobacteriaceae dominate the core microbiome and contribute to the resistome of arugula (Eruca sativa Mill.). Microbiome. 2019;7:13.

6. González-Plaza JJ, Blau K, Milaković M, Jurina T, Smalla K, Udiković-Kolić N. Antibiotic-manufacturing sites are hot-spots for the release and spread of antibiotic resistance genes and mobile genetic elements in receiving aquatic environments. Environ Int. 2019:130:104735.

7. Lopatto E, Choi J, Colina A, Ma L, Howe A, Hinsa-Leasure S. Characterizing the soil microbiome and quantifying antibiotic resistance gene dynamics in agricultural soil following swine CAFO manure application. PloS One. 2019; 14:e0220770.

8. Karimi B, Maron PA, Boure NC-P, Bernard N, Gilbert D, Ranjard L. Microbial diversity and ecological networks as indicators of environmental quality. Environ Chem Lett. 2017;15:265-81.

9. Langdon A, Crook N, Dantas G. The effects of antibiotics on the microbiome throughout development and alternative approaches for therapeutic modulation. Genome Med. 2016;8:39.

10. Jernberg C, Löfmark S, Edlund C, Jansson JK. Long-term impacts of antibiotic exposure on the human intestinal microbiota. Microbiology. 2010; 156:3216-23.

11. Yassour M, Vatanen $T$, Siljander $H$, Hämäläinen $A-M$, Härkönen $T$, Ryhänen SJ, et al. Natural history of the infant gut microbiome and impact of antibiotic treatment on bacterial strain diversity and stability. Sci Transl Med. 2016;8:343ra81.

12. Mahnert A, Moissl-Eichinger C, Zojer M, Bogumil D, Mizrahi I, Rattei T, et al. Man-made microbial resistances in built environments. Nat Commun. 2019; 10:1-12.

13. Obermeier MM, Wicaksono WA, Taffner J, Bergna A, Poehlein A, Cernava T, et al. Plant resistome profiling in evolutionary old bog vegetation provides new clues to understand emergence of multi-resistance. ISME J. 2020:1-17. https://doi.org/10.1038/s41396-020-00822-9.

14. Grube M, Cernava T, Soh J, Fuchs S, Aschenbrenner I, Lassek C, et al. Exploring functional contexts of symbiotic sustain within lichen-associated bacteria by comparative omics. ISME J. 2015;9:412-24.

15. Pennisi E. A lichen ménage à trois; 2016.

16. Fernández-Mendoza F, Fleischhacker A, Kopun T, Grube M, Muggia L. ITS 1 metabarcoding highlights low specificity of lichen mycobiomes at a local scale. Mol Ecol. 2017;26:4811-30.

17. Cernava T, Aschenbrenner IA, Grube M, Liebminger S, Berg G. A novel assay for the detection of bioactive volatiles evaluated by screening of lichenassociated bacteria. Front Microbiol. 2015;6:398.

18. Cernava T, Erlacher A, Aschenbrenner IA, Krug L, Lassek C, Riedel K, et al. Deciphering functional diversification within the lichen microbiota by metaomics. Microbiome. 2017:5:82

19. Conti ME, Cecchetti G. Biological monitoring: lichens as bioindicators of air pollution assessment-a review. Environ Pollut. 2001;114:471-92.

20. Cernava T, Vasfiu Q, Erlacher A, Aschenbrenner IA, Francesconi K, Grube M, et al. Adaptions of lichen microbiota functioning under persistent exposure to arsenic contamination. Front Microbiol. 2018:9:2959.

21. Gries C. Lichens as indicators of air pollution. In: Nash III TH (ed) Lichen biology. Cambridge University Press, Cambridge. 1996:240-254.

22. Bialvaei $A Z$, Samadi $\mathrm{KH}$. Colistin, mechanisms and prevalence of resistance. Curr Med Res Opin. 2015;31:707-21.

23. Chopra I, Roberts M. Tetracycline antibiotics: mode of action, applications, molecular biology, and epidemiology of bacterial resistance. Microbiol Mol Biol Rev. 2001;65:232-60.

24. Kusstatscher P, Cernava T, Liebminger S, Berg G. Replacing conventional decontamination of hatching eggs with a natural defense strategy based on antimicrobial, volatile pyrazines. Sci Rep. 2017;7:1-8.

25. Mbanaso F, Coupe S, Charlesworth S, Nnadi E, Ifelebuegu A. Potential microbial toxicity and non-target impact of different concentrations of glyphosate-containing herbicide $(\mathrm{GCH})$ in a model pervious paving system Chemosphere. 2014;100:34-41.

26. Motta EV, Raymann K, Moran NA. Glyphosate perturbs the gut microbiota of honey bees. Proc Natl Acad Sci. 2018;115:10305-10.

27. Paterson DL, Isler B, Stewart A. New treatment options for multiresistant gram negatives. Curr Opin Infect Dis. 2020;33:214-23.
28. Xia X, Wang Z, Fu Y, Du X, Gao B, Zhou Y, et al. Association of colistin residues and manure treatment with the abundance of mcr-1 gene in swine feedlots. Environ Int. 2019;127:361-70.

29. Ye G, Qiu Y, He X, Zhao L, Shi F, Lv C, et al. Effect of two macrocephala flavored powder supplementation on intestinal morphology and intestinal microbiota in weaning pigs. Int J Clin Exp Med. 2015;8:1504.

30. Semedo M, Song B, Sparrer T, Phillips RL. Antibiotic effects on microbial communities responsible for denitrification and $\mathrm{N} 2 \mathrm{O}$ production in grassland soils. Front Microbiol. 2018;9:2121.

31. Mbanaso F, Coupe S, Charlesworth S, Nnadi E. Laboratory-based experiments to investigate the impact of glyphosate-containing herbicide on pollution attenuation and biodegradation in a model pervious paving system. Chemosphere. 2013;90:737-46.

32. Krug L, Erlacher A, Berg G, Cernava T. A novel, nature-based alternative for photobioreactor decontaminations. Sci Rep. 2019;9:1-10.

33. Köberl M, Müller H, Ramadan EM, Berg G. Desert farming benefits from microbial potential in arid soils and promotes diversity and plant health PLoS One. 2011;6:e24452.

34. Lieber A, Kiesel B, Babel W. Microbial diversity analysis of soil by SSCP fingerprinting technique using TGGE Maxi System. In: Prozessregulation in der Rhizosphäre. Vieweg+Teubner Verlag, Wiesbaden. 2003:61-5.

35. Parada AE, Needham DM, Fuhrman JA. Every base matters: assessing smal subunit rRNA primers for marine microbiomes with mock communities, time series and global field samples. Environ Microbiol. 2016;18:1403-14.

36. Walters W, Hyde ER, Berg-Lyons D, Ackermann G, Humphrey G, Parada A, et al. Improved bacterial 16S rRNA gene (V4 and V4-5) and fungal internal transcribed spacer marker gene primers for microbial community surveys. Msystems. 2016;1:e00009-15.

37. Bolyen E, Rideout JR, Dillon MR, Bokulich NA, Abnet CC, Al-Ghalith GA, et al Reproducible, interactive, scalable and extensible microbiome data science using QIIME 2. Nat Biotechnol. 2019;37:852-7.

38. Martin M. Cutadapt removes adapter sequences from high-throughput sequencing reads. EMBnet J. 2011;17:10-2.

39. Callahan BJ, McMurdie PJ, Rosen MJ, Han AW, Johnson AJA, Holmes SP. DADA2: high-resolution sample inference from Illumina amplicon data. Nat Methods. 2016:13:581.

40. Rognes T, Flouri T, Nichols B, Quince C, Mahé F. VSEARCH: a versatile open source tool for metagenomics. PeerJ. 2016:4:e2584

41. Pruesse E, Quast C, Knittel K, Fuchs BM, Ludwig W, Peplies J, et al. SILVA: a comprehensive online resource for quality checked and aligned ribosomal RNA sequence data compatible with ARB. Nucleic Acids Res. 2007;35:7188-96.

42. Bolger AM, Lohse M, Usadel B. Trimmomatic: a flexible trimmer for Illumina sequence data. Bioinformatics. 2014;30:2114-20.

43. Menzel P, Ng KL, Krogh A. Fast and sensitive taxonomic classification for metagenomics with Kaiju. Nat Commun. 2016;7:1-9.

44. Nurk S, Meleshko D, Korobeynikov A, Pevzner PA. metaSPAdes: a new versatile metagenomic assembler. Genome Res. 2017;27:824-34.

45. Langmead B, Salzberg SL. Fast gapped-read alignment with Bowtie 2. Nat Methods. 2012;9:357.

46. Buchfink $B$, Xie $C$, Huson DH. Fast and sensitive protein alignment using DIAMOND. Nat Methods. 2015:12:59.

47. Huerta-Cepas J, Szklarczyk D, Forslund K, Cook H, Heller D, Walter MC, et al. eggNOG 4.5: a hierarchical orthology framework with improved functional annotations for eukaryotic, prokaryotic and viral sequences. Nucleic Acids Res. 2016:44:D286-93.

48. Arango-Argoty G, Garner E, Pruden A, Heath LS, Vikesland P, Zhang L. DeepARG: a deep learning approach for predicting antibiotic resistance genes from metagenomic data. Microbiome. 2018;6:1-15.

49. Looft T, Johnson TA, Allen HK, Bayles DO, Alt DP, Stedtfeld RD, et al. In-feed antibiotic effects on the swine intestinal microbiome. Proc Natl Acad Sci. 2012;109:1691-6.

50. Rascovan N, Telke A, Raoult D, Rolain JM, Desnues C. Exploring divergent antibiotic resistance genes in ancient metagenomes and discovery of a novel beta-lactamase family. Environ Microbiol Rep. 2016;8:886-95.

51. Liao Y, Smyth GK, Shi W. featureCounts: an efficient general purpose program for assigning sequence reads to genomic features. Bioinformatics. 2014;30:923-30.

52. McMurdie PJ, Holmes S. phyloseq: an R package for reproducible interactive analysis and graphics of microbiome census data. Plos One. 2013;8:e61217.

53. Oksanen J, Kindt R, Legendre P, O'Hara B, Stevens MHH, Oksanen MJ, et al. The vegan package. Community Ecol Package. 2007;10:631-7. 
54. Chong J, Liu P, Zhou G, Xia J. Using MicrobiomeAnalyst for comprehensive statistical, functional, and meta-analysis of microbiome data. Nat Protoc. 2020;15:799-821.

55. Dhariwal A, Chong J, Habib S, King IL, Agellon LB, Xia J. MicrobiomeAnalyst: a web-based tool for comprehensive statistical, visual and meta-analysis of microbiome data. Nucleic Acids Res. 2017:45:W180-8.

56. Segata N, lzard J, Waldron L, Gevers D, Miropolsky L, Garrett WS, et al. Metagenomic biomarker discovery and explanation. Genome Biol. 2011;12:1-18.

57. Kassambara A. ggpubr:"ggplot2" based publication ready plots. R Package Version 01; 2017. p. 6.

58. Hembach N, Schmid F, Alexander J, Hiller C, Rogall ET, Schwartz T. Occurrence of the mcr-1 colistin resistance gene and other clinically relevant antibiotic resistance genes in microbial populations at different municipal wastewater treatment plants in Germany. Front Microbiol. 2017:8:1282.

59. Martínez JL. Antibiotics and antibiotic resistance genes in natural environments. Science. 2008;321:365-7.

60. Van Goethem MW, Pierneef R, Bezuidt OK, Van De Peer Y, Cowan DA Makhalanyane TP. A reservoir of 'historical'antibiotic resistance genes in remote pristine Antarctic soils. Microbiome. 2018;6:40.

61. Martinez JL, Sánchez MB, Martínez-Solano L, Hernandez A, Garmendia L, Fajardo $\mathrm{A}$, et al. Functional role of bacterial multidrug efflux pumps in microbial natural ecosystems. FEMS Microbiol Rev. 2009;33:430-49.

62. Fierer N, Leff JW, Adams BJ, Nielsen UN, Bates ST, Lauber CL, et al. Crossbiome metagenomic analyses of soil microbial communities and their functional attributes. Proc Natl Acad Sci. 2012;109:21390-5.

63. Kurenbach B, Marjoshi D, Amábile-Cuevas CF, Ferguson GC, Godsoe W, Gibson P, et al. Sublethal exposure to commercial formulations of the herbicides dicamba, 2, 4-dichlorophenoxyacetic acid, and glyphosate cause changes in antibiotic susceptibility in Escherichia coli and Salmonella enterica serovar Typhimurium. MBio. 2015;6:e00009-15.

64. Van Bruggen A, He M, Shin K, Mai V, Jeong K, Finckh M, et al. Environmental and health effects of the herbicide glyphosate. Sci Total Environ. 2018;616: 255-68

65. Rybakova D, Cernava T, Köberl M, Liebminger S, Etemadi M, Berg G. Endophytes-assisted biocontrol: novel insights in ecology and the mode of action of Paenibacillus. Plant Soil. 2016:405:125-40.

66. Silva-Junior EA, Ruzzini AC, Paludo CR, Nascimento FS, Currie CR, Clardy J, et al. Pyrazines from bacteria and ants: convergent chemistry within an ecological niche. Sci Rep. 2018;8:1-7.

67. Raymann K, Shaffer Z, Moran NA. Antibiotic exposure perturbs the gut microbiota and elevates mortality in honeybees. PLoS Biol. 2017;15: e2001861.

68. Raymond F, Ouameur AA, Déraspe M, lqbal N, Gingras H, Dridi B, et al. The initial state of the human gut microbiome determines its reshaping by antibiotics. ISME J. 2016;10:707-20.

69. Liu Y-Y, Wang Y, Walsh TR, Yi L-X, Zhang R, Spencer J, et al. Emergence of plasmid-mediated colistin resistance mechanism MCR-1 in animals and human beings in China: a microbiological and molecular biological study. Lancet Infect Dis. 2016:16:161-8.

70. Maciuca El, Cummins ML, Cozma A, Rimbu C, Guguianu E, Panzaru C, et al. Genetic features of mcr-1 mediated colistin resistance in CMY-2-producing Escherichia coli from Romanian poultry. Front Microbiol. 2019;10:2267.

71. Sun J, Zhang H, Liu Y-H, Feng Y. Towards understanding MCR-like colistin resistance. Trends Microbiol. 2018;26:794-808.

72. Simon C, Daniel R. Metagenomic analyses: past and future trends. Appl Env Microbiol. 2011;77:1153-61.

73. Cabral DJ, Wurster Jl, Belenky P. Antibiotic persistence as a metabolic adaptation: stress, metabolism, the host, and new directions. Pharmaceuticals. 2018;11:14.

74. Li J, Xie S, Ahmed S, Wang F, Gu Y, Zhang C, et al. Antimicrobial activity and resistance: influencing factors. Front Pharmacol. 2017;8:364.

75. Cernava T, Aschenbrenner IA, Soh J, Sensen CW, Grube M, Berg G. Plasticity of a holobiont: desiccation induces fasting-like metabolism within the lichen microbiota. ISME J. 2019;13:547-56.

76. Francino M. Antibiotics and the human gut microbiome: dysbioses and accumulation of resistances. Front Microbiol. 2016:6:1543.

77. Ahmadjian V. Lichens are more important than you think. BioScience. 1995; 45:124.

78. You Y, Silbergeld EK. Learning from agriculture: understanding lowdose antimicrobials as drivers of resistome expansion. Front Microbiol. 2014;5:284.
79. Van Boeckel TP, Brower C, Gilbert M, Grenfell BT, Levin SA, Robinson TP, et al. Global trends in antimicrobial use in food animals. Proc Natl Acad Sci. 2015;112:5649-54.

80. Bengtsson-Palme J, Kristiansson E, Larsson DJ. Environmental factors influencing the development and spread of antibiotic resistance. FEMS Microbiol Rev. 2018:42:fux053.

\section{Publisher's Note}

Springer Nature remains neutral with regard to jurisdictional claims in published maps and institutional affiliations.
Ready to submit your research? Choose BMC and benefit from:

- fast, convenient online submission

- thorough peer review by experienced researchers in your field

- rapid publication on acceptance

- support for research data, including large and complex data types

- gold Open Access which fosters wider collaboration and increased citations

- maximum visibility for your research: over $100 \mathrm{M}$ website views per year

At BMC, research is always in progress.

Learn more biomedcentral.com/submissions 